\title{
Oxidative Stress in Controlled Hypotension: Assessment with a Novel Oxidative Stress Marker
}

\author{
Cihan Doger ${ }^{\circledR}$, Ayca Tuba Dumanli Ozcan ${ }^{1}$, Ezgi Erkilic1 ${ }^{\circledR}$, Suleyman Ellik ${ }^{\circledR}$, Semsi Mustafa Aksoy ${ }^{3}$, Murat Alisik ${ }^{4}$, \\ Ozcan Erel ${ }^{5}$ \\ ${ }^{1}$ Ankara City Hospital, Universiteler M. Bilkent, Ankara, Turkey \\ ${ }^{2}$ Ankara Ulucanlar Ophtalmology Education and Research Hospital, Department of Anesthesiology and Reanimation, Ankara, Turkey \\ ${ }^{3}$ Yıldırım Beyazıt University, Faculty of Medicine, Department of Anesthesiology, Ankara,Turkey \\ ${ }^{4}$ Ankara City Hospital, Department of Medical Biochemistry, Ankara, Turkey \\ ${ }^{5}$ Yıldırım Beyazıt University, Faculty of Medicine, Department of Medical Biochemistry, Ankara,Turkey
}

Correspondence Author: Ayca Tuba Dumanli Ozcan

E-mail: draycaozcan@gmail.com

Received: $22.09 .2020 \quad$ Accepted: 08.02.2021

\begin{abstract}
Objective: The aim of this study was to evaluate the effects of oxidative stress on thiol-disulfide homeostasis caused by hypotensive anesthesia in mastoidectomy-tympanoplasty cases undergoing controlled hypotension.

Methods: Fifty adult patients scheduled for mastoidectomy and tympanoplasty were included in the study. Anesthesia was induced with lidocaine, propofol, rocuronium, and remifentanil. The maintenance of anesthesia was continued with remifentanil infusion (target mean arterial pressure as $60-65 \mathrm{mmHg}$ ) along with $2 \%$ sevoflurane/40\% O2/air mixture. Blood samples were taken 5 times at the t0 (before induction), $\mathrm{t} 1$ (intraoperatively after intubation), $\mathrm{t} 2$ (first hour) and $\mathrm{t} 3$ (second hour of the operation) and t4 (following recovery). Total thiol (TT) and Native Thiol (NT) levels were measured, and thus, Di-Sulphide (SS), Di-Sulphide/Native Thiol (SSNT), Di-Sulphide/Total Thiol (SSTT), and Native Thiol/ Total Thiol (NTTT) values were estimated.

Results: During the operation, progressive decrease was observed in thiol levels of patients. There was a significant decrease in $\mathrm{t} 3$ thiol values when compared with t0 value. Thiol values were observed to have returned to baseline values after recovery from anesthesia ( $p>0.05$ ). SS, SSNT and SSTT levels were found as increased in $\mathrm{t} 1$ blood samples, but increase in SSNT and SSTT levels was significant. Throughout the operation, values were observed to have dropped and reverted back to initial values.

Conclusion: Since the measurement of thiol-disulfide blood values is able to show the instantaneous state of oxidative stress, it can be used in anesthesia practice in which every event occurs very quickly.
\end{abstract}

Keywords: Thiol-disulfide homeostasis, controlled hypotension, oxidative stress.

\section{INTRODUCTION}

The plasma thiol pool comprises organic compounds containing sulfhydryl group called mercaptans which consists mainly of albumin and protein thiols. In addition, cysteine (Cys), cysteinylglycine, glutathione, homocysteine and $\gamma$-glutamylcysteine known as low molecular-weight thiols contribute to the thiol pool (1). Thiol-containing proteins present in the cell play an important role in redox-sensitive reactions (2). Thiols react with oxidizing agents to form thioldisulfide bonds (3). These disulfide bonds are again reduced to thiol groups when oxidative stress conditions change. Thus, dynamic thiol-disulfide homeostasis is maintained. Dynamic thiol-disulfide homeostasis has an important role in antioxidant protection, detoxification, apoptosis, regulation of enzymatic activities, and cellular signaling mechanisms (4). Thiol disulfide balance was demonstrated to change in such diseases as cardiovascular disease, cancer, and rheumatoid arthritis. This balance is known to chance in numerous diseases and conditions apart from them (5).
Plasma thiol levels are mostly measured using Ellman's reagent, 4,4'-dithiodipyridine (4-DPS), or by adopting more complex methods. However, these methods are not suitable for use in automated analyzers. Erel and Neselioglu have developed a new automated method that measures thioldisulfide homeostasis (5). This new method allows the evaluation of both thiol [total thiol (TT), native thiol (NT)] and disulfide (SS) side of the reaction. The increase in disulfide [disulfide/native thiol ratio (SSNT), disulfide/total thiol ratio (SSTT)] together with the decrease in the amount of thiol refers to the increase in oxidation. In the contrary case, it may be said that reduction increases. It was projected that determining how much thiol-disulfide values change under which conditions would shed light on the pathogenesis of many diseases, and numerous studies have been carried out on this subject $(6,7)$.

Controlled hypotension is a technique used to increase surgical visibility during surgery and to reduce surgical 
complications. It is aimed to keep intraoperative mean arterial pressure between $50-65 \mathrm{mmHg}$ or systolic arterial pressure between $80-90 \mathrm{mmHg}$ or $30 \%$ of the input value (8). Volatile anesthetics, sympathetic antagonists, sodium nitroprusside, nitroglycerine hydralazine, trimethaphan, adenosine, phenoldopam, $\alpha-2$ agonists, and some opioid analgesics may be used for the purpose of controlled hypotension. Remifentanil is an opioid analgesic and is a $\mu$-opioid receptor agonist. Due to being metabolized by non-specific esterases having very fast clearance, and its provision of rapid recovery, it is often used in the clinics for the purpose of controlled hypotension (8). However, inadvertent formation of free oxygen radicals is possible during hypotension application (7). Thiol-disulfide homeostasis can be affected very rapidly from oxidative changes, and oxygen radicals are likely to alter hemostasis. In cases where hypoxia or ischemia is possible, blood thiol-disulfide levels of the patient provide valuable information about the state of oxidative stress $(9,10)$. The aim of this study was to evaluate the effects of oxidative stress caused by hypotensive anesthesia on thiol-disulfide homeostasis in mastoidectomy-tympanoplasty cases undergoing controlled hypotension with remifentanil.

\section{METHODS}

\subsection{Hypothesis and Number of Patients}

The study hypothesis $\left(\mathrm{H}_{1}\right)$ was established as "controlled hypotension creates a significant change in patients' blood thiol and disulfide levels." Since the change of blood thiol and disulfide levels of patients were a dependent variable based on initial value (t0), there was no control group in this study. In other words, since the measured thiol-disulfide blood values were compared with the to value, the to value constituted the control group.

As a result of power analysis (gPower 3.1.9.2, Franz Paul, Universitat Kiel, 2014, Germany) conducted by assuming that $20 \%$ change in time between time slices in blood thiol and disulfide levels was considered to be significant by using study data obtained from normal volunteers [5], it was estimated that the study should include 49 patients (Effect Size 0.5, $\alpha=0.05,1-\beta=0.82$ ). Fifty patients were planned to be included in the study by taking into consideration the patients that may be excluded from the study due to unforeseen issues.

\subsection{Patient Group}

The study was conducted following the approval of the Ankara Atatürk Training and Research Hospital Clinical Research Ethics Committee (Approval Date: 14.1.2015, No: 26379996/03). Written informed consents were obtained from the patients included in the study. Adult patients undergoing mastoidectomy and tympanoplasty surgery in ASA I risk group were included in the study. Patients that had any abnormal values in their preoperative routine examinations, patients with known comorbidities, and patients that had continuous drug use were not included in the study. Patients with difficult intubation during anesthesia, allergies or intraoperative acute cardiac or respiratory problems, and other unpredictable anesthesia-related complications were determined as exclusion criteria. The patients were evaluated in the anesthesia outpatient clinic one day before the operation. Patients without any abnormal findings were included in the study.

\subsection{Anesthesia Practice}

After the patients were taken to the operation room, routine monitoring (5-lead ECG, Pulse Oximeter, and non-invasive arterial blood pressure cuff) and intravenous (IV) access with an appropriate catheter were provided. Intraoperative fluid requirement was satisfied with crystalloid $0.9 \% \mathrm{NaCl}$ solution as $8-10 \mathrm{ml} / \mathrm{kg}$ by also evaluating the fasting status of the patients. Lidocaine $1 \mathrm{mg} / \mathrm{kg}$, Propofol $3 \mathrm{mg} / \mathrm{kg}$, rocuronium $0.5 \mathrm{mg} / \mathrm{kg}$ and remifentanil $1 \mu \mathrm{g} / \mathrm{kg}$ were administered intravenously for induction of anesthesia following $3 \mathrm{~min}$ preoxygenation. Remifentanil infusion was commenced after induction and continued by gradually increasing until the target mean arterial pressure (MAP) value reached to 60-65 $\mathrm{mmHg}(0.125-0.5 \mu \mathrm{g} / \mathrm{kg} / \mathrm{min})$. All patients underwent invasive arterial blood pressure monitoring with a $22 \mathrm{G}$ cannula from the radial artery (after the Allen test) to ensure frequent drawing of blood and to carry out strict blood pressure monitoring. The maintenance of anesthesia was continued with remifentanil infusion along with $2 \%$ sevoflurane/40\% 02/air mixture. Ventilation was ensured with an end-tidal $\mathrm{CO} 2\left(\mathrm{ETCO}_{2}\right)$ pressure of $32-36 \mathrm{mmHg}$. In the event that the heart rate drops below $50 / \mathrm{min}, 0.5 \mathrm{mg}$ of atropine IV was administered. Mean arterial pressure values that continued below $60 \mathrm{mmHg}$ for 1 minute and above were accepted as hypotension, remifentanil dosage would be halved, and if it would not reach normal values within 5 minutes, it was stopped till mean arterial pressure would reach normal values, and ephedrine $5 \mathrm{mg}$ IV was administered. Remifentanil infusion was continued when normal mean arterial pressure was maintained. For postoperative analgesia remifentanil infusion was terminated with contramal $50 \mathrm{mg}$ IV close to the end of the case (10 min before). The patient was extubated abiding by extubation criteria and was taken to the recovery unit. An intraarterial catheter was withdrawn in the recovery unit and patient was sent to the ward when Aldrete score was sufficient.

\subsection{Blood Sampling, Analysis and Interpretation of Results}

A total of 5 blood samples were collected, namely, before induction (t0) in the preoperative operation room, after intraoperative intubation ( $\mathrm{t} 1$ ), at the 1 st hour (t2), at the 2nd hour $(t 3)$, and after recovery (when Aldrete score $=10)(t 4)$. Blood samples were centrifuged at 3600rpm for 10 minutes. Serum/plasma samples were separated and stored at $-80^{\circ} \mathrm{C}$. Total thiol (TT) and native thiol (NT) levels were measured simultaneously in all blood samples. Disulfide (SS), disulfide/ native thiol (SSNT), disulfide/total thiol (SSTT) and native 
thiol/total thiol (NTTT) values were also calculated using these parameters. Tests were evaluated by a new technique developed by Prof. Dr. Ozcan Erel (5) and were interpreted by him. Cost of kits and tests were covered by Prof. Dr. Ozcan Erel.

\subsection{Collection of Data}

Age, sex, height, body weight values of patients, duration of anesthesia, duration of surgery, and blood samples obtained at $\mathrm{t} 0, \mathrm{t} 1, \mathrm{t} 2, \mathrm{t} 3$, and $\mathrm{t} 4$ time slices were collected as anonymous data. Collected data were recorded using Excel software (Excel 2010@ for Windows v14.0.7, Microsoft Corporation).

\subsection{Statistical Analysis}

The distribution of the data of each parameter was evaluated by Shapiro-Wilk test. The data showed normal distribution. Age, height, body weight data were given in tables by calculating mean and standard deviation. Paired-T test was used to compare TT, SS, NT, NTTT, SSTT, and SSNT values with $\mathrm{t} 0$ value at $\mathrm{t} 1, \mathrm{t} 2, \mathrm{t} 3$, and $\mathrm{t} 4$ time slices. $\mathrm{p}<0.05$ was considered significant. SPSS 23 software (2015, IBM SPSS Statistics for Windows, Version 23.0. Armonk, NY: IBM Corp.) was used for statistical estimations.

\section{RESULTS}

A total of 50 patients in ASA I risk group were included in the study. One patient was excluded from the study due to the fact that the targeted mean arterial pressure values could not be reached during the operation. The mean age of the patients was $37.0 \pm 10.7$ years, and $57.1 \%$ of the patients were male. BMI measurements were 26.0 \pm 4 .2. Demographic data of patients are given in Table 1.

Table 1. Demographic data of patients

\begin{tabular}{|l|l|l|}
\hline Parameter & & Value \\
\hline Age (year) & & $37.0 \pm 10.7$ \\
\hline Gender* & Male & $28(57.1)$ \\
\hline & Female & $21(42.9)$ \\
\hline Height $(\mathrm{cm})$ & & $169.5 \pm 7.0$ \\
\hline Weight $(\mathrm{kg})$ & & $74.9 \pm 13.4$ \\
\hline BMI $\left(\mathrm{kg} / \mathrm{m}^{2}\right)$ & & $26.0 \pm 4.2$ \\
\hline
\end{tabular}

Values are presented as mean \pm standard deviation of the parameters; *values are presented as number and percent of cases [n (\%)]; BMI: body mass index.

None of the patients included in the study had difficult intubation and any complications of surgery and anesthesia. The mean duration of anesthesia was $119.1 \pm 37.1$ minutes, and the duration of surgery was $94.5 \pm 33.2$ minutes. Since the anesthesia time of all patients was 60 minutes or more, t0, t1, t2, and t4 samples could be obtained. However, t3 blood samples were obtained from 25 patients with anesthesia duration of 120 minutes or above. Medications were administered to all patients without any issues within the study protocol, and MAP, ETCO2, and other vital signs were kept at the desired values. None of the patients required Atropine, but $4(8.2 \%)$ patients required the administration of $5 \mathrm{mg}$ ephedrine.

There was a progressive decrease in TT and NT values of patients at all times during the operation (Figure 1), and when all measurement values were compared with t0 values, there was a significant decrease in $\mathrm{t3}$ TT, NT, and SS levels. Thiol values more or less returned to baseline values following recovery ( $p>0.05)$. The change of TT, NT, SS, SSTT, SSNT, and NTTT values of patients throughout the operation is given in Table 2.

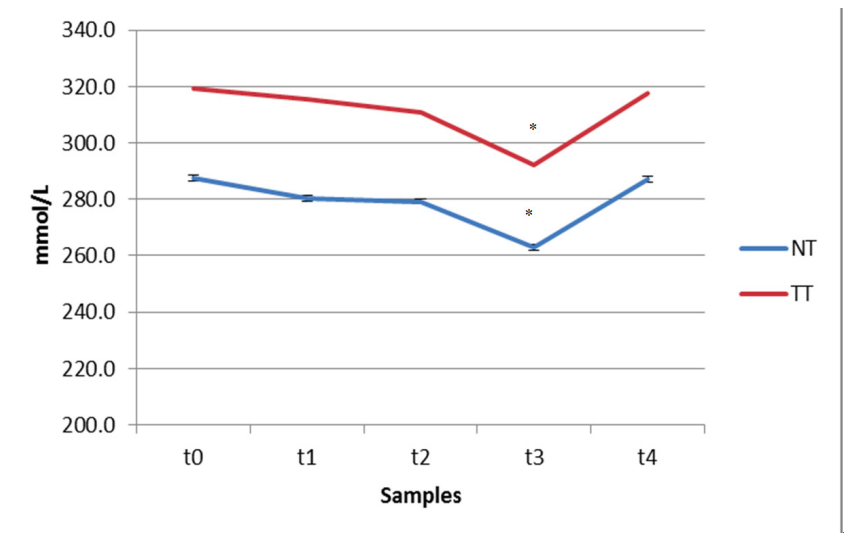

Figure 1. The change in TT and NT values of the blood samples of patients taken during the operation. NT: Native Thiol, TT: Total Thiol, t0: before anesthesia, $t 1$ : after intubation, $t 2: 1$ st hour, $t 3: 2$ nd hour, t4: after anesthesia, * $p<0.05$ statistically significant, $t 3-t 0:$ paired T-test

Table 2. The change of TT, NT, SS, SSTT, SSNT, and NTTT values of patients throughout the operation

\begin{tabular}{|l|l|l|l|l|l|}
\hline & to $(\mathrm{n}=49)$ & t1 $(\mathrm{n}=49)$ & t2 $(\mathrm{n}=49)$ & $\mathrm{t} 3(\mathrm{n}=25)$ & $\mathrm{t} 4(\mathrm{n}=49)$ \\
\hline $\mathrm{TT}(\mu \mathrm{mol} / \mathrm{L})$ & $319.4 \pm 84.1$ & $315.7 \pm 65.6$ & $310.9 \pm 84.1$ & $292.2 \pm 59.8^{*}$ & $317.8 \pm 72.6$ \\
\hline $\mathrm{NT}(\mu \mathrm{mol} / \mathrm{L})$ & $287.6 \pm 75.1$ & $280.4 \pm 60.8$ & $279.3 \pm 78.2$ & $263.0 \pm 54.0^{*}$ & $287.1 \pm 71.1$ \\
\hline $\mathrm{SS}(\mu \mathrm{mol} / \mathrm{L})$ & $15.9 \pm 8.3$ & $17.6 \pm 7.0$ & $15.8 \pm 7.3$ & $14.6 \pm 7.6^{*}$ & $15.3 \pm 6.0$ \\
\hline $\mathrm{SSTT}(\mu \mathrm{mol} / \mathrm{L})$ & $4.8 \pm 2.1$ & $5.6 \pm 2.1^{*}$ & $5.2 \pm 2.2$ & $5.0 \pm 2.4$ & $5.0 \pm 2.3$ \\
\hline $\mathrm{SSNT}(\mu \mathrm{mol} / \mathrm{L})$ & $5.5 \pm 2.6$ & $6.4 \pm 2.7^{*}$ & $5.9 \pm 2.8$ & $5.7 \pm 3.0$ & $5.7 \pm 3.0$ \\
\hline $\mathrm{NTTT}(\mu \mathrm{mol} / \mathrm{L})$ & $90.3 \pm 4.1$ & $88.9 \pm 4.2^{*}$ & $89.7 \pm 4.4$ & $90.1 \pm 4.8$ & $90.0 \pm 4.6$ \\
\hline
\end{tabular}

TT: Total Thiol, NT: Native Thiol, SS: Disulfide, , SSTT: Disulfide/Total Thiol, SSNT: Disulfide/Native Thiol, NTTT: Native Thiol/Total Thiol

It was established in $\mathrm{t} 1$ blood samples that mean blood SS levels of patients had an increase, and that these levels started to decrease throughout the operation. The increase in t1 SS values was not statistically significant when compared with baseline values, but the decrease in $\mathrm{t} 3$ value was found as significant (Figure 2). It was observed that mean SSNT and SSTT rates in $\mathrm{t} 1$ blood samples increased, and that the values decreased and went back to the initial values during the operation. The increase after anesthesia induction was found as statistically significant $(p<0.05)$ (Figures 3 and 4). 


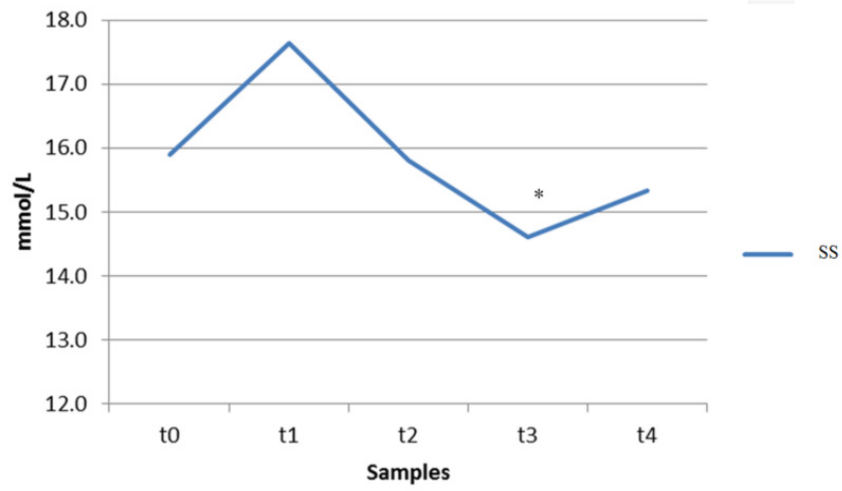

Figure 2. The change in SS values of the blood samples of patients taken during the operation. SS: Disulfide, t0: before anesthesia, t1: after ilntubation, t2: 1st hour, t3: 2nd hour, t4: after anesthesia., * $p<0.05$ statistically significant, $t 3$-t0:paired $T$-test

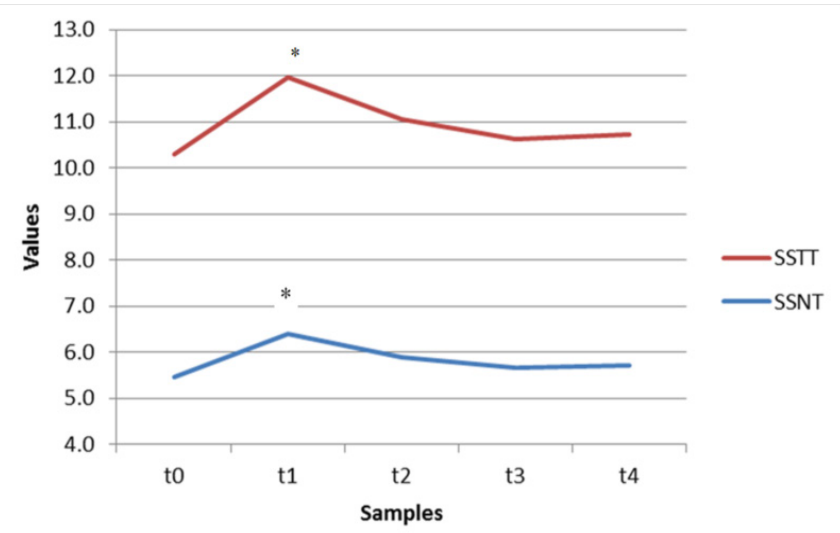

Figure 3. The change in SSNT and SSTT rates of the patients throughout the operation. SSNT: (Disulfide/Native Thiol) $\times 100$ SSTT:(Disulfide/Total Thiol)x100, t0: before anesthesia, t1: after intubation, t2: 1st hour, t3: 2nd hour, t4: after anesthesia, ${ }^{*} p<0.05$ statistically significant, t1-t0: paired T-test

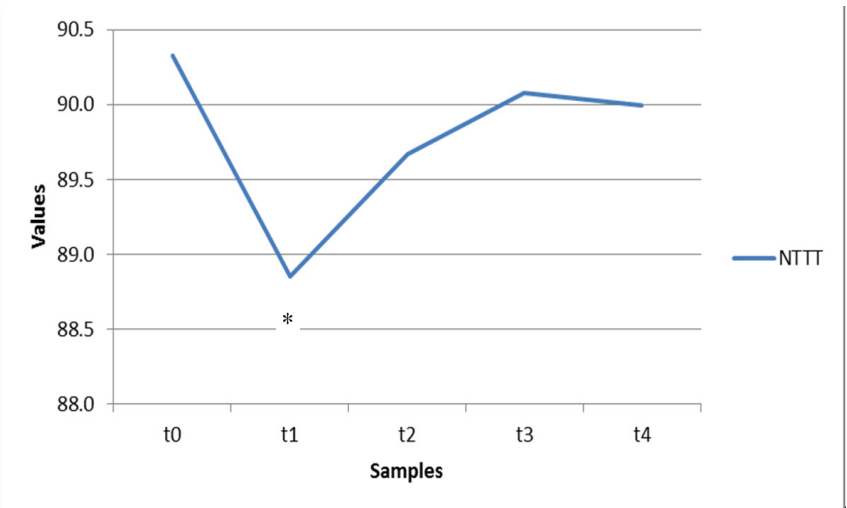

Figure 4. The change in NTTT rates of the patients throughout the operation. NTTT: Native Thiol/Total Thiol)x100, t0: before anesthesia, t1: after intubation, t2: 1st hour, t3: 2nd hour, t4: after anesthesia, values are presented as mean of the parameters, ${ }^{*} p<0.05$ statistically significant, t1-t0:paired T-test

\section{DISCUSSION}

In our study in which we evaluated the effects of controlled hypotension on dynamic thiol homeostasis reflecting biochemical evaluation of oxidative stress changes, we established thiol-disulfide blood changes accordant with stress factors. In our literature review, we did not find any study examining the effects of controlled hypotension on thiol balance. Therefore, since articles that can be taken as a reference for the evaluation of the results are nonexistent, we assessed our results in the light of studies on the effects of various diseases, general anesthesia, and other interventions on thiol-disulfide balance.

Thiols acting as fast electron acceptors have negative standard reduction potential. When an oxidant interacts with a thiol group, it is neutralized to a relatively less toxic byproduct than the oxidized thiol. Plasma TT (-SH+-S-S-), NT (-SH), and SS (-SS-) levels are more frequently used for routine diagnoses and follow-ups of numerous diseases and metabolic syndromes (5). Thiol-disulfide deposition may occur in extremely oxidative situations. Increased disulfide levels were often associated with increased oxidative stress states (diabetes mellitus, atherosclerosis, cancer, hypertension, etc.) (11-13). Similarly, in their study, Topuz et al. found that thiol-disulfide mechanism plays an important role in acute pulmonary embolism conditions (14).

Controlled hypotension in tympanoplasty cases is a frequently used method for reducing blood loss and providing better visibility in the surgical field. However, controlled hypotension can disrupt the microcirculatory autoregulation by suppressing the autonomic nervous system, thereby leading to end organ damage (15). However, there are no studies on mortality and morbidity except for the very old studies on this subject (16). Disruption of microcirculatory autoregulation may cause oxidative stress in addition to anesthesia and surgical stress response $(17,18)$. Oxidative stress plays an important role in the pathogenesis of numerous diseases. Therefore, the importance of thioldisulfide balance increases. Thiol disulfide values measured by manual and difficult methods have become very easy thanks to the automated method developed by Erel et al. (5). Thiol balance is a dynamic process that can manifest long-term pathologies as well as detecting momentary abnormalities. In our study, we showed that thiol and disulfide values changed significantly during intubation process. It is a correct approach to benefit from dynamism of thiol-disulfide tests in the field of anesthesia in which bleeding and ischemia, and thus, oxidative stress.

The rapid change of thiol and disulfide blood levels creates difficulty in determining normal values. In our study, we found to thiol values close to normal values established by Erel et al. (5) but slightly lower in the preoperative period. We believe that this may be due to thiol value change stemming from preoperative stress, despite our patient group comprised of patients that had no health issues other than the surgical intervention that they would undergo. 
Anesthesia triggers inflammatory processes during surgery. The emergence of inflammatory mediators and free radicals also causes membrane damage of peroxidation products (19). Anesthetic agents used in studies have been reported to affect oxidative stress (20-22). In our study, we avoided using thiol containing sodium thiopental as anesthesia induction agent in order not to affect laboratory values, and we opted for using propofol that decreases oxidative stress by reducing lipid peroxidation $(23,24)$. The balance between prooxidants and antioxidants in our body may be temporarily disrupted by volatile anesthetic agents. Studies have shown that sevoflurane is converted to free oxygen radicals by the enzyme CYP2E1 $(25,26)$. Sevoflurane enhances lipoperoxidation in vivo and in vitro by inhibiting antioxidant enzymes (27). In addition to these studies, in their study that evaluated the oxidant and antioxidant effects of sevoflurane, desflurane, and propofol infusions in patients undergoing laparoscopic cholecystectomy, Erbas et al. found the antioxidant effects of sevoflurane and propofol as significantly higher (21).

In this study, a statistically significant increase was established in SSNT and SSTT values of patients after intubation. In our literature review, we found that pre-oxygenation conducted with $100 \%$ oxygen during induction period was reported to cause an increase in oxidative stress (28). We also preoxygenated our patients for 3 minutes during induction of anesthesia. In addition, it may be suggested that the formation of inflammatory and catabolic state through stress response during endotracheal intubation contribute to the increase in oxidation, leading to a rise in SS level. In the following periods of the operation, the graph of the SS level was interpreted as oxidative stress continuing albeit in a diminishing manner. When SSNT and SSTT elevation are evaluated in detail, it is observed that the increase in SS level and the decrease in NT level are effective. Our study showed that SS level increased rapidly and NT level decreased in case of stress.

In their study that evaluated thiol-disulfide balance of various pressures and albumin modified by ischemia in 36 child patients that would undergo laparoscopic surgery, Ozgunay et al. (29) did not find any difference in thiol-disulfide balance but established the albumin values that ischemia modified as high. In another study conducted by Polat et al. (30) on laparoscopic surgery, a significant decrease was established in NT, TT, and SS levels during operation. They did not establish any change in SSNT and SSTT levels. Unlike the study by Ozgunay, they did not find any statistically significant difference in ischemia-modified albumin levels. In their study that evaluated open surgery and laparoscopic surgery in previous hernia repair, Polat et al. established changes in sulfhydryl levels in both groups. TT, NT, and SS levels also manifested a decrease (t3) in our study. Unlike the above, increase was observed in SSTT and SSSNT levels in the blood sample drawn at the 11 time in our study. As explained above, post-intubation is associated with the occurrence of TT and NT decrease and SS value increase. Significant decrease of NTTT in t1 suggests that NT is instantaneously affected from oxidative stress. Long-term evaluation showed that SS, TT, and NT values continued to decrease in patients that underwent controlled hypotension. In our study group, since the mean duration of anesthesia was 119.1 \pm 37.1 minutes, it was not possible to evaluate the actual effects of controlled hypotension. However, when the graphs are evaluated, it may be foreseen that thiol and disulfide values would decrease in long-term anesthesia.

Due to the fact that there was no previous study on the same subject and that thiol-disulfide blood levels could be affected by numerous individual factors, no control group was formed. As a control group, it was assumed that the change would be more significant compared to preoperative blood values of patients. The ability of anesthesia agents that does not directly affect thiol-disulfide balance to indirectly reduce or increase oxidation makes it difficult to fully understand the effects of controlled hypotension.

\section{CONCLUSION}

We evaluated in our study the change in thiol and disulfide blood values in tympanoplasty cases that underwent controlled hypotension. We found that the stress response after intubation increased SSNT and SSTT levels and decreased NTTT. We predicted that SSNT, SSTT, and NTTT values can be used in rapidly developing conditions. In addition, we found that NT, TT, and SS values decreased progressively during controlled hypotension. We showed that oxidative stress increased as the duration of controlled hypotension is prolonged. Since the measurement of thioldisulfide blood values is able to show the instantaneous state of oxidative stress, it can be used in anesthesia practice where every event occurs very quickly.

\section{REFERENCES}

[1] Turell L, Radi R, Alvarez B. The thiol pool in human plasma: The central contribution of albumin to redox processes. Free Radic Biol Med 2013;65:244-253.

[2] Moran LK, Gutteridge JM, Quinlan GJ. Thiols in cellular redox signaling and control. Curr Med Chem 2001;8: 763-772.

[3] Cremers $\mathrm{CM}$, Jakob U. Oxidant sensing by reversible disulfide bond formation. J Biol Chem 2013;288: 26489-26496.

[4] Biswas S, Chida AS, Rahman I. Redox modifications of proteinthiols: emerging roles in cell signaling. Biochem Pharmacol 2006; 71: 551-564.

[5] Erel O, Neselioglu S. A novel and automated assay for thiol/ disulphide homeostasis. Clin Biochem 2014;47: 326-332.

[6] Kaman A, Aydin Teke T, Gayretli Aydin ZG, Karacan Kucukali G, Neselioglu S, Erel O, Tanır G. How does dynamic thiol/ disulphide homeostasis work in the pathogenesis of Kawasaki disease? Pediatr int 2019;61: 913-918.

[7] Agan V, Celik H, Eren MA, Agan FZ, Erel O, Neselioglu S, Koyuncu I, Gonel A. An Investigation of Oxidative Stress and Thiol/Disulphide Homeostasis in Graves' Disease. Medicina 2019; 55(6):275.

[8] Degoute CS. Controlled hypotension: a guide to drug choice. Drugs 2007;67:1053-1076. 
[9] Karatas M, Ozis TN, Buyuksekerci M, Gunduzoz M, Ozakinci OG, Gok G, Neşelioğlu, S, Erel, Ö. Thiol-disulfide homeostasis and ischemia-modified albumin levels as indicators of oxidative stress in welders' lung disease. Hum Exp Toxicol 2019;38:12271234.

[10] Maksimova MY, Ivanov AV, Virus ED, Alexandrin VV, Nikiforova KA, Bulgakova PO, Ochtova FR, Suanova ET, Piradov MA, Kubatiev AA. Disturbance of thiol/disulfide aminothiols homeostasis in patients with acute ischemic stroke stroke: Preliminary findings. Clin Neurol Neurosurg 2019;183:105393.

[11] Gur M, Elbasan Z, Yildiray Sahin D, Yildiz Koyunsever N, Seker T, Ozaltun B, Caylı M, Kocyigit A. DNA damage and oxidative status in newly diagnosed, untreated, dipper and non-dipper hypertensive patients. Hypertens Res 2013;36(2):166-171.

[12] Kundi H, Erel O, Balun A, Cicekcioglu H, Cetin M, Kiziltunc E, Neşelioğlu S, Topçuoğlu C, Örnek E. Association of thiol/ disulfide ratio with syntax score in patients with NSTEMI. Scand Cardiovasc J 2015;49(2):95-100.

[13] Go YM, Jones DP. Cysteine/cystine redox signaling in cardiovascular disease. Free Radic Biol Med. 2011;50: 495509.

[14] Topuz M, Kaplan M, Akkus O, Sen O, Yunsel HD, Allahverdiyev S, Erel O, Koc M, Gur M. The prognostic importance of thiol/ disulfide homeostasis in patients with acute pulmonary thromboembolism. Am J Emerg Med 2016;34: 2315-2319.

[15] Degoute CS, Ray MJ, Manchon M, Dubreuil C, Banssillon V. Remifentanil and controlled hypotension; comparison with nitroprusside or esmolol during tympanoplasty. Can J Anaesth 2001;48 :20-27.

[16] Hampton LJ, Little DM, Jr. Complications associated with the use of controlled hypotension in anesthesia. AMA Arch Surg 1953; 67:549-556.

[17] 17.Degoute CS, Ray MJ, Gueugniaud PY, Dubreuil C. Remifentanil induces consistent and sustained controlled hypotension in children during middle ear surgery. Can J Anaesth 2003; 50:270-276.

[18] Fodale V, Schifilliti D, Pratico C, Santamaria LB. Remifentanil and the brain. Acta Anaesthesiol Scand 2008;52:319-326.

[19] Koksal GM, Sayilgan C, Aydin S, Uzun H, Oz H. The effects of sevoflurane and desflurane on lipid peroxidation during laparoscopic cholecystectomy. Eur J Anaesthesiol 2004;21:217220.

[20] Schilling T, KozianA, SenturkM, Huth C, ReinholdA, Hedenstierna
G, Hachenberg T. Effects of volatile and intravenous anesthesia on the alveolar and systemic inflammatory response in thoracic surgical patients. Anesthesiology 2011;115:65-74.

[21] Erbas M, Demiraran Y, Yildirim HA, Sezen G, Iskender A, Karagoz I, Kandis H. Comparison of effects on the oxidant/antioxidant system of sevoflurane, desflurane and propofol infusion during general anesthesia. Rev Bras Anestesiol 2015;65 :68-72.

[22] Ozcan ATD, Doger C, Ozturk L, Yungul A, Kurtsahin $M$, Neselioglu S, Ergin M, But A. Comparison of the Effects of Sevoflurane and Desflurane on Thiol-Disulfide Homeostasis in Patients Undergoing Laparoscopic Cholecystectomy. Eurasian J Med 2019;51: 70-74.

[23] Li Volti G, Basile F, Murabito P, Galvano F, Di Giacomo C, Gazzolo D, Vadalà S, Azzolina R, D'Orazio N, Mufeed H, Vanella L, Nicolosi A, Basile G, Biondi A. Antioxidant properties of anesthetics: the biochemist, the surgeon and the anesthetist. Clin Ter 2008; 159 :463-469.

[24] Tsuchiya M, Sato EF, Inoue M, Asada A. Open abdominal surgery increases intraoperative oxidative stress: can it be prevented? Anesth Analg 2008;107:1946-1952.

[25] Kevin LG, Novalija E, Riess ML, Camara AK, Rhodes SS, Stowe DF. Sevoflurane exposure generates superoxide but leads to decreased superoxide during ischemia and reperfusion in isolated hearts. Anesth Analg 2003; 96 :949-955.

[26] Wong CH, Liu TZ, Chye SM, Lu FJ, Liu YC, Lin ZC, Chen CH. Sevoflurane-induced oxidative stress and cellular injury in human peripheral polymorphonuclear neutrophils. Food Chem Toxicol 2006;44:1399-1407.

[27] Sato N, Fujii K, Yuge O. In vivo and in vitro sevoflurane induced lipid peroxidation in guinea-pig liver microsomes. Pharmacol Toxicol 1994;75:366-370.

[28] Habre W, Peták F. Perioperative use of oxygen: variabilities across age. Br J Anaesth 2014;113 Suppl 2:ii26-36.

[29] Ozgunay SE, Ustundag Y, Karasu D, Uguz I, Erel O, Korfali G, Kaya M. The Effect of Different Intraabdominal Pressures On Thiol/Disulfide Homeostasis in Children Who Underwent Ambulatory Laparoscopic Surgery: a Prospective Randomized Study. J Laparoendosc Adv Surg Tech A 2018;28:1142-1147.

[30] Polat C, Kahraman A, Yilmaz S, Koken T, Serteser M, Akbulut G, Arikan Y, Dilek ON, Gokce O. A comparison of the oxidative stress response and antioxidant capacity of open and laparoscopic hernia repairs. J Laparoendosc Adv Surg Tech A 2003;13(3):167-173. 